\title{
What XiaoHongShu Users Care About: An analysis of Online Review Comments
}

\author{
Wenru Tian ${ }^{1, *}, \dagger$ Yiyang $\mathrm{Xiao}^{2, \dagger}$ Longkun $\mathrm{Xu}^{3, \dagger}$ \\ ${ }^{1}$ Department of Mathematic Science, Tsinghua University, 100089, Beijing, China \\ ${ }^{2}$ School of Economics \& Trade, Hunan University, 410006, Changsha, China \\ ${ }^{3}$ State University of New York at Buffalo, 14260, Buffalo, America \\ *Corresponding author.Email: twr17@mails.tsinghua.edu.cn \\ These authors contributed equally.
}

\begin{abstract}
This paper studies the attributes of XiaoHongShu, a social e-commerce business, by analyzing the big data set of user comments through data mining and sentiment analysis to find out the problems existing in XiaoHongShu and put forward suggestions. Findings show that the main attributes of XiaoHongShu can be summarized into three aspects: social media, sharing platform, and software service. Besides, many users have negative attitudes to their experience in XiaoHongShu. This research can help XiaoHongShu further explore the platform's value and bring a better experience to users. It can also provide more information and reference for the exploration of social e-commerce.
\end{abstract}

Keywords: XiaoHongShu, User experience, Online reviews, Sentiment analysis.

\section{INTRODUCTION}

In recent years, increasing platforms in China have adopted a new form of fusion of social media and ecommerce transactions called social e-commerce. Social e-commerce, based on social networks, promotes commodity transactions through UGC (user-generated content), social interaction, and other ways to socialize ecommerce, thus helping the development of e-commerce [1]. XiaoHongShu is one of the most typical examples of community e-commerce. From 2013 to 2014, XiaoHongShu completed the transformation from sharing community to e-commerce, the operation form of XiaoHongShu gradually appears to be embryonic. The platform is mainly divided into two sections: social sharing community and shopping mall. Users can not only find beauty skills, clothes matching, home decoration, travel sharing, and even learning experiences in the social sharing community, but also conduct overseas online shopping in the "Welfare Club", which is a shopping mall of XiaoHongShu, thus completing the business closed-loop [2]. As of January 2020, the market value of XiaoHongShu has exceeded 20 billion yuan [3]. By August, the registered users of XiaoHongShu have exceeded 450 million, and the monthly active users have exceeded 100 million [4].
Many researchers take XiaoHongShu as an example to explore the field of social e-commerce. Existing studies have investigated XiaoHongShu's community marketing [5], operation model [6], development strategy [7], factors that affect XiaoHongShu's user loyalty [3], and satisfaction [8], as well as user consumption status [9]. Existing studies show that the trust established by the actual user word of mouth in the sharing community of XiaoHongShu improves the platform's conversion rate and user activity and loyalty, giving XiaoHongShu an advantage difficult for other e-commerce companies to replicate [2]. By contrast, the disadvantage of $\mathrm{XiaoHongShu}$ is that it is a platform transformed into ecommerce in the later stage, so the development of the shopping mall is not perfect, and there are threats from other competitors [6]. The current research is mainly focused on the mall section of XiaoHongShu, and indepth analysis and recommendations are provided. For example, researchers suggest improving the after-sales service of XiaoHongShu's shopping mall, expanding the supply chain and sales channels of products in the mall, and seeking the best cooperation mode [6]. However, little study has explored the complex problems of XiaoHongShu from the perspective of customer experience.

This study investigates the current issues in XiaoHongShu by analyzing the big data set of online 
comments of XiaoHongShu users to put forward pertinent suggestions to help XiaoHongShu improve its functions and attract more users. This paper mainly uses text mining and sentiment analysis to analyze online comments. The text-mining results show three attributes of XiaoHongShu: social media, sharing platform, and software service. Next, it can be identified that the positive and negative opinions of XiaoHongShu users through sentiment analysis so that this study can find out more accurately the aspects of XiaoHongShu that need improvement, thus helping us to put forward more reasonable suggestions. The contribution of this paper lies in two parts. Firstly, by analyzing various factors affecting the user experience of community e-commerce, XiaoHongShu can fully play the respective values of community and e-commerce more effectively, which is conducive to providing a better experience for users XiaoHongShu. Secondly, this study can provide valuable information for the exploration in community ecommerce and provide a reference for the homogenous platform [3].

\section{METHODOLOGY}

\subsection{Data collection}

The data set is from the KuChuan website (https://www.kuchuan.com), and 21,743 online review comments are collected from 2020/07/05 to 2021/07/05. Needed to be stated, the comments obtained are from the Apple Store users, while in some Android stores, the phenomenon of "water army" or "paid posters" (a group of Internet ghostwriters paid to post online comments with particular content) is prominent; also some comments may have default text or default positive comments, which in turn increases the number of comments that need to be sieved, reducing efficiency.

Then in the analysis, the comments of different stars are divided into different databases and imported into python to remove some comments containing only the individual word, such as "good", "great", "egregious", etc., so as to eliminate invalid comments, duplicate comments or malicious comments. After that, this research counts the word frequency and forms the word cloud map.

\subsection{Data analysis}

Data analysis involves three stages. In the first phase, text mining is performed to determine the semantic insights of the comments, and the words are categorized to help us do further research [10]. In the second phase, by viewing and text mining the official update text of XiaoHongShu within one month after the update time, this paper evaluates the content of the XiaoHongShu app update. This verification is necessary and meaningful because "update" is mentioned many times as a word in the word frequency and the word cloud shown in the next section, indicating that users are highly interested in reviews and will comment on the updated version, especially those with more critical content [11]. For example, the blind box did not appear as a topic or concept, but in the New Year update of XiaoHongShu 6.80 , "collect the blind box, share 300 million" was promoted as a significant update for the New Year. This phenomenon suggests that some major updates are not reflected by the same word when analyzing the user evaluation of XiaoHongShu, while often having a tremendous subsequent impact on the user experience. In the last stage, sentiment analyses are used to determine users' opinions about XiaoHongShu. This study calculates the proportion of positive comments among all the comments containing keyword A and use the proportion to represent users' positive or negative attitudes towards keyword A. Once the favorable comment ratio is less than $50 \%$, users are generally dissatisfied with the keyword, and XiaoHongShu needs to adjust and improve it appropriately.

\section{RESULTS AND DISCUSSION}

\subsection{Data description}

Figure 1 shows that 7041 reviews are only one star, 841 reviews are two stars, 1201 reviews are three stars, 1247 reviews are four stars, and 11413 reviews are five stars among the data.

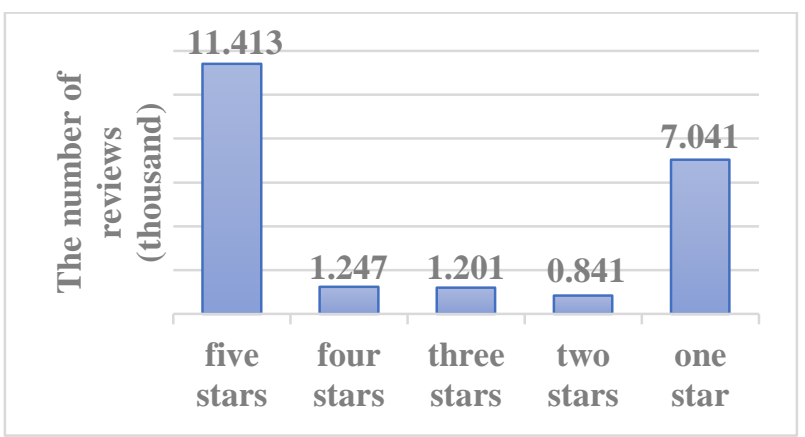

Figure 1 The number of reviews with different star ratings.

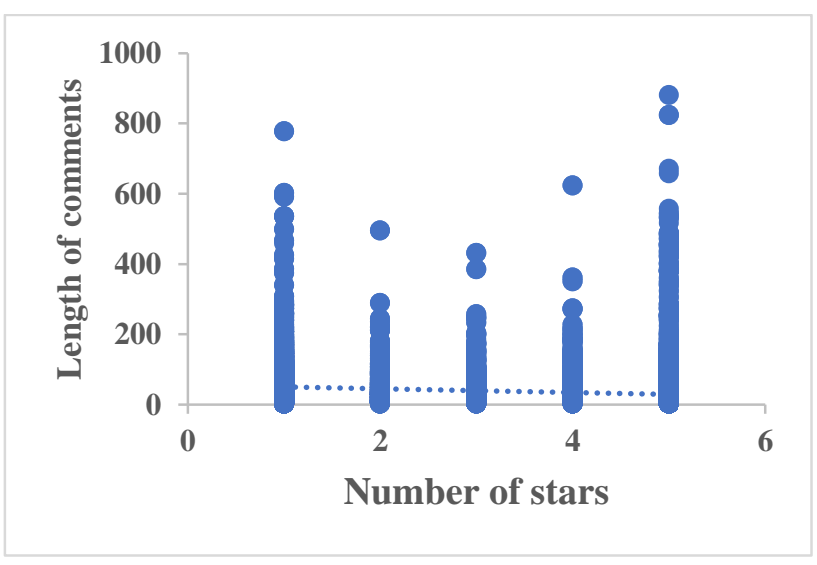

Figure 2 Distribution of the word length 
After filtering and removing shorter, meaningless, and repetitive text, 20,264 reviews are obtained. Figure2 shows that the average length of reviews is about 34.85 words, the average length of reviews with one star is 49.10 , two stars is 46.72 , three stars is 42.85 , four stars is 43.35 , and the average length of five stars is 27.36 , showing that the users in express their dissatisfaction with the use of the XiaoHongShu software state more content. In contrast, the use of words in the highest rating of five stars is less. Overall, most comments are under 200 words, $69 \%$ of which are 10 to 100 words, with only a very small percentage (1.48\%) of comments exceeding 200 words in length.

\subsection{Text mining results}

As shown in Table 1, the main keywords in the text mining process all fall into the three main attributes of XiaoHongShu discussed previously: social software, sharing platform, and software service. This paper also discusses three sections separately in the following.

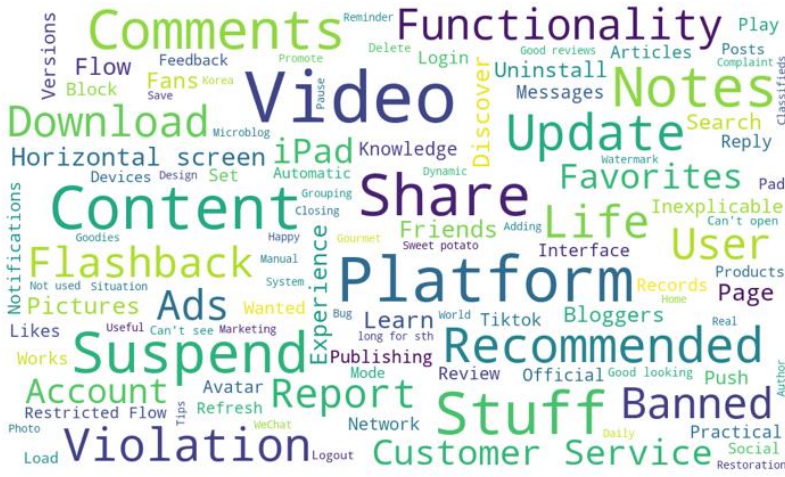

Figure 3 The word cloud of XiaoHongShu

Figure 3 above is a visual display of the words mentioned most frequently in users' comments. Users attach importance to social-related words such as "user" or" friends"; the user also pays attention to the XiaoHongShu as a sharing platform, shown by the words "video," content," etc., at the same time, software services of XiaoHongShu are highlighted through words such as "functionality", "Ads" etc. The above is the reason why this research has divided the keywords into three categories (Social media, Sharing platform, and Software service) in the table below.

Table 1 Word frequency statistics of comments

\begin{tabular}{|c|c|c|c|c|c|}
\hline Social media & & ig platform & & Software service & \\
\hline words & frequency & words & frequency & words & frequency \\
\hline Suspend & 1193 & Video & 1973 & Update & 956 \\
\hline Comment & 1146 & Platform & 1476 & Functionality & 713 \\
\hline Violation & 801 & Content & 1349 & Download & 694 \\
\hline Ban & 714 & Stuff & 1288 & Flashback & 671 \\
\hline User & 703 & Share & 1250 & Ads & 645 \\
\hline Report & 625 & Notes & 1163 & Customer service & 587 \\
\hline Proclaim & 511 & Recommend & 931 & Horizontal screen & 543 \\
\hline Friends & 480 & Life & 783 & Pages & 469 \\
\hline Fans & 396 & Favorite & 551 & Experience & 460 \\
\hline Boggers & 377 & find & 489 & Version & 345 \\
\hline Messages & 328 & Learn & 472 & Interface & 304 \\
\hline Reply & 282 & Flowing & 456 & Network & 298 \\
\hline Block & 277 & Photo & 456 & Play & 293 \\
\hline Avatar & 271 & Search & 372 & Set & 283 \\
\hline Socialize & 196 & Post & 349 & Official & 272 \\
\hline Delete & 186 & Knowledge & 321 & Refresh & 242 \\
\hline Write off & 185 & Record & 300 & Device & 208 \\
\hline Drop out & 179 & Release & 298 & Feedback & 204 \\
\hline WeChat & 158 & Pragmatic & 297 & Mode & 200 \\
\hline Subset & 149 & Wanting & 293 & Loading & 200 \\
\hline
\end{tabular}




\subsubsection{Social media}

The software to achieve socializing through the Internet is social. The most used social media globally are Facebook, WhatsApp, Messenger, and WeChat. The social attributes of XiaoHongShu, compared to the products of the above Internet giants, are not as prominent.

As is shown in Table 1, the top ten keywords are "suspend, comments, violations, ban, users, report, proclaim, friends, fans, boggers", from which it is clear that the social aspects of XiaoHongShu, mainly under the user's post, the users' mutual comments, rather than instant messaging. Furthermore, the word "report", "block", and so on reveal that the software presents part of the unfriendly social environment. Under the role of review environment and social mechanism, XiaoHongShu does not need users to frequently open for social relationship maintenance, inducing the number of daily online users and related indicators are not outstanding.

\subsubsection{Sharing platform}

XiaoHongShu App takes video, text, and pictures as the communication carrier, which is a way to record and share one's life and experience on the platform, and meet the psychological demand of users' daily interaction. The top ten keywords are video, platform, content, stuff, share, notes, recommend, life, favorite, and find, showing that users value what others share on the platform and use it in their work and study. It is no surprise that the word "recommend" has emerged, as mostly sharing platforms now have their algorithmic recommendation models at the product level. This tech ensures that XiaoHongShu, a content production platform, has a constant flow of highquality content production.

However, when further analyzing the comments with the words video and content, it has been found that many users mentioned the concept of "plagiarism" but did not use the same description, so there is no high word frequency, for example:

"I am outraged because my hard-working notes were no longer be recommended due to the transcription by others."

"Theft of pictures is disgusting, and the official simply ignore a few words to cope with the line as the original author of the stolen pictures is hard to bear."

Therefore, as a sharing platform, the XiaoHongShu has severely discouraged the original creators, making the source and foundation of content on which the platform relies to survive a blow.

\subsubsection{Software service}

In the aspect of software services, the top ten keywords are "update, functionality, download, flashback, Ads, customer service, horizontal screen, pages, experience, version." Users' comments reflect a solid suggestion orientation in the category of leisure tools, i.e., users' comments reflect aspects of XiaoHongShu that they feel dissatisfied with, such as the app's flashback and ads and the horizontal screen problem of the iPad version of the XiaoHongShu app. For every app, user experience is crucial, which is why the intense competition in the internet market.

\subsection{The research of the updates}

This research counted 127 updates in the last two years (2019.07.14-2021-07-14) on the update details page of XiaoHongShu on KuChuan's website, and through text analysis, only 10 of them have "unique" detailed update descriptions, and the remaining 117 are "optimize product experience and fix some issues."YiYang Xiao is responsible for writing the methodology, completing the text analysis and update research of Little Red XiaoHongShu reviews, and giving relevant research results and recommendations; Among the ten updates, this paper selects the updates on January 11, 2020 (version 6.29). The updated text of version 6.29 is--

"On January 21, come to the XiaoHongShu to shake out your own top signature! Collect five kinds of good luck small red stick, the first five prizes to share a big red envelope! Nine days of Spring Festival, shake the small red stick, divide a billion!'

Table 2 Word frequency statistics of comments of version 6.29 within a month

\begin{tabular}{|c|c|c|c|}
\hline One star & & Five star & \\
\hline words & frequency & words & frequency \\
\hline Partition & 18 & Game & 157 \\
\hline Ads & 16 & worthy & 34 \\
\hline Video & 15 & Mastery & 32 \\
\hline Activity & 12 & Player & 17 \\
\hline One billion & 10 & Fun & 16 \\
\hline Collection & 10 & Liver & 16 \\
\hline Billions & 9 & BGM & 16 \\
\hline Propagate & 8 & Picture & 16 \\
\hline Fake & 7 & Strategy & 15 \\
\hline Word game & 6 & Treasurebox & 10 \\
\hline
\end{tabular}


A total of 676 user comments one month after its update time is used to conduct a similar text mining analysis, but at this stage of the analysis, one-star and five-star ratings $(84.05 \%)$ with the highest degree of representation are used, and the high word frequency words related to the updated content are obtained.

Obviously, due to the positioning of XiaoHongShu, the words related to games and money appearing in the reviews have a strong correlation with the activities in the updated content of the software. The keywords appearing in the top five in the one-star reviews are partition, ads, video, activity, one billion; the top five in the five-star reviews are game, worthy, mastery, player, fun. Although the words appearing in the negative reviews seem to be more relevant to the activity, their frequency is not high. In general, it is supposed that users who did not get the expected benefits from the campaign expressed their dissatisfaction with different game details through their comments, while users who were satisfied with the campaign just gave ordinary positive comments as usual.

This activity is an attempt to improve users' stickiness in the last part of the "social media" property, through daily activities, to improve the interaction between users, increase the number of times users open the software, and at the same time enhance the influence of the software.

\subsection{Sentiment analysis}

Table 3 is the sentiment analysis of XiaoHongShu, which shows that XiaoHongShu, as a social platform for knowledge sharing and interactive dating, has won the general recognition of its users. However, most of them negatively affect the platform functions, such as video playback, content audit, customer service, etc. For example, the word with the highest frequency is "Video", but only $42 \%$ of all reviews containing "Video" are positive. Users have a generally negative attitude towards XiaoHongShu's video playback function. Most negative comments are related to the video's pause, speed, and push mechanism. This is where XiaoHongShu can improve in the future

As a social platform, XiaoHongShu does meet the purpose of sharing life and making friends, but the functional defects of the platform seriously affect the user's sense of experience. The main reasons that lead to negative comments are as follows: 1. Content push 2 . Content audit mechanism; 3. Advertising; 4. Suspend; 5. Customer service. These five points are related to the core functions of the XiaoHongShu, which are deeply linked with the platform income and user experience.
Table 3 Likelihood scores of sentiment analysis

\begin{tabular}{|c|c|c|c|}
\hline Concept & Count & Likelihood & Sentiment \\
\hline Knowledge & 321 & $86 \%$ & Positive \\
\hline Socialize & 196 & $71 \%$ & Positive \\
\hline Pragmatic & 297 & $67 \%$ & Positive \\
\hline Friends & 480 & $53 \%$ & Positive \\
\hline Share & 1250 & $48 \%$ & Negative \\
\hline Platform & 1476 & $46 \%$ & Negative \\
\hline Video & 1973 & $41 \%$ & Negative \\
\hline Wanting & 293 & $40 \%$ & Negative \\
\hline Record & 300 & $39 \%$ & Negative \\
\hline Interface & 304 & $30 \%$ & Negative \\
\hline Fans & 396 & $24 \%$ & Negative \\
\hline Avatar & 271 & $22 \%$ & Negative \\
\hline Content & 1349 & $19 \%$ & Negative \\
\hline Official & 272 & $13 \%$ & Negative \\
\hline Notes & 1163 & $11 \%$ & Negative \\
\hline Messages & 328 & $11 \%$ & Negative \\
\hline Feedback & 204 & $8 \%$ & Negative \\
\hline WeChat & 158 & $8 \%$ & Negative \\
\hline Mode & 200 & $6 \%$ & Negative \\
\hline Network & 298 & $5 \%$ & Negative \\
\hline Refresh & 242 & $3 \%$ & Negative \\
\hline Loading & 200 & $3 \%$ & Negative \\
\hline Play & 293 & $2 \%$ & Negative \\
\hline Suspend & 1193 & $1 \%$ & Negative \\
\hline Violation & 801 & $1 \%$ & Negative \\
\hline Ban & 714 & $1 \%$ & Negative \\
\hline Flashback & 671 & $1 \%$ & Negative \\
\hline
\end{tabular}

\subsection{Suggestions}

\subsubsection{Establish benign interaction to improve social media functions}

Establish relevant disciplinary and reward measures for users who frequently and maliciously comment on others, give a particular time ban, and gradually aggravate; In contrast, for users who maintain the commenting atmosphere for a long time and output highquality comments, they can be given certain honorary and material rewards to form a whole social platform to advocate good comments and protect the experience of users in daily use.

\subsubsection{Strengthen content audit, combat plagiarism, and improve UGC quality}

The essence of XiaoHongShu is a life-sharing platform, and users generally recognize its social function, but the defects of the platform and the quality of UGC cannot reach the proper standard of adequate socialization. In the subsequent improvement, the "platform function" and "content" should be the core. For duplicated, plagiarized, and carried content from other platforms, its traffic should be restricted or deleted, and reward and punishment mechanisms to protect original authors and support originality should be introduced, such as for enhancing the promotion of original content and labeling its tweets with corresponding labels for users to identify. In addition, XiaoHongShu should 
strengthen the content audit and content pushing mechanism, review the notes posted by users, avoid vulgar and harmful content from appearing on users' homepages, and maintain the ecological balance XiaoHongShu community.

\subsubsection{Improve software services and enhance user stickiness}

Consumers' perceptions of products and services ultimately come down to their experiences, which is the basis for evaluating products and services. An excellent sensory experience and interactive experience of an app will not bring significant satisfaction. So the development of XiaoHongShu should pay attention to improving user experience in all aspects of the user. First, pay attention to the design of the product interface. Many users are "sensual animals"; they pay attention to the first impression left by the product, whether the interface is aesthetic, whether the style is generous and straightforward without losing functional integrity, whether the color is distinct and natural, etc.; second, improve the way the product service. Product services should be closely linked to users' needs because companies produce products to meet the diverse needs of users, such as the above-mentioned iPad version, horizontal screen function, download function, etc. Third, increase the openness of the product. XiaoHongShu will be closely linked with some other social ways, which can increase users' convenience and expand the functions of XiaoHongShu to build the XiaoHongShu into an open platform. In short, social software openers should improve users' experience in all aspects in various ways to enhance users' stickiness.

\section{CONCLUSION}

This paper found the existing problems of XiaoHongShu and gave the corresponding suggestions by studying the attributes of XiaoHongShu through data mining and sentiment analysis. The research results show that the attributes of XiaoHongShu can be summarized as social media, sharing platform, and software service. From these three attributes, what the users of XiaoHongShu care about can be understood. Therefore, according to these attributes, this paper puts forward the following suggestions respectively: 1) Add chat rooms or discussion groups to the platform to improve social functions. 2) Add more quality and diverse content to keep users engaged and active. 3) Improving user experience by optimizing software functions, perfecting the suitability of software on each device, or promoting the feedback mechanism and other details. In addition, Sentiment analysis shows that XiaoHongShu users have negative attitudes towards many aspects of their experience, such as content push, content audit mechanism, advertising, etc. XiaoHongShu can optimize these aspects more pertinently. This study can help
XiaoHongShu improve its functions and bring a better experience to users. It can also provide helpful information and reference for other platforms in the field of social e-commerce.

\section{REFERENCES}

[1] Fan Yuqing. Research on User Behavior of Social Ecommerce Platform.2019. Shandong Normal University,MA Thesis.

[2] Wang Lin. A Case Study of XiaoHongShu on User Behavior Analysis and Countermeasures of Social and E-business Platform. Modern Marketing (Academics Edition) $\quad\{3\} .05(2021): 80-83$. doi:10.19932/j.cnki.22-1256/ f.2021.05.080.

[3] Cao Shan. Research on Factors Influencing User Loyalty of Community E-Commerce.2019. South China University of Technology,MA Thesis.

[4] Julia xu jiao rong, and Pan Honggang. "platform under the background of economic social electric business platform for the XiaoHongShu marketing strategy research, for example." modern business. 27 (2020) : 35 and 36, doi: 10.14097 / j.carol carroll nki. 5392/2020.27.014

[5] Wang E P, et al." A study on community commodity sales: A case study of "XiaoHongShu" APP." Industry \& Technology Forum 20.07(2021):76-77. doi:CNKI:SUN: CyYT.0.2021-07-035.

[6] Jeremy. "the operation mode of XiaoHongShu." new media studies (2021) : 7.0942 to 45. Doi: 10.16604 / j.carol carroll nki issn2096-0360.2021.09.013.

[7] Jia Li. Development Strategy of Social App XiaoHongShu. Western Leather 43.05(2021):103105. doi:CNKI: Sun: xbpG.0.2021-05-053.

[8] QiuXue, can, sun and Wu Kaixin. "based on customer perceived value of the research to the influential factors of customer satisfaction, in the XiaoHongShu, for instance." China quotient theory. 6 (2021): 44-48, doi: 10.19699 / j.carol carroll nki issn2096-0298.2021.06.044.

[9] RaoYan. "under the new media environment, network consumption present condition analysis and guidance, in the XiaoHongShu, for example." the Chinese market. 02 (2021): 129-130. The doi: 10.13939 / j.carol carroll nki ZGSC. 2021.02.129.

[10] Cheng, M., 2017. Big data management, Fausto Pedro García Márquez and Benjamin Lev. Inf. Technol. Tour. 17 (4), 455-456.

[11] Liesch, P.W., Håkanson, L., McGaughey, S.L., Middleton, S., Cretchley, J., 2011. The evolution of the international business field: a scientometric investigation of articles published in its premier journal. Scientometrics 88 (1), 17-42. 